Review began 08/15/2021 Review ended 08/24/2021 Published 08/27/2021

\section{(c) Copyright 2021}

Sheikh et al. This is an open access article distributed under the terms of the Creative Commons Attribution License CC-BY 4.0 . which permits unrestricted use, distribution, and reproduction in any medium, provided the original author and source are credited.

\title{
Comparing Pain Intensity Using the Numeric Rating Scale and Defense and Veterans Pain Rating Scale in Patients Revisiting the Emergency Department
}

Sophia Sheikh ${ }^{1}$, Jennifer Fishe ${ }^{2}$, Ashley Norse ${ }^{1}$, Morgan Henson ${ }^{1}$, Divya James ${ }^{3}$, Warren Sher $^{1}$, Michelle Lott ${ }^{1}$, Colleen Kalynych ${ }^{1}$, Phyllis Hendry ${ }^{1}$

\begin{abstract}
1. Emergency Medicine, University of Florida College of Medicine - Jacksonville, Jacksonville, USA 2. Pediatric Emergency Medicine, University of Florida College of Medicine - Jacksonville, Jacksonville, USA 3. Emergency
\end{abstract} Medicine, University of Florida College of Medicine, Gainesville, USA

Corresponding author: Sophia Sheikh, sophia.sheikh@jax.ufl.edu

\section{Abstract}

\section{Objective}

To determine the relationship between Numeric Rating Scale (NRS) and Defense and Veterans Pain Rating Scale (DVPRS) as pain intensity measures, we compared pain scores to sociodemographic and treatment data in patients revisiting the emergency department (ED).

\section{Methods}

After Institutional Review Board approval, 389 adults presenting within 30 days of an index visit were enrolled. Pain scores were classified as follows: 0-3 (mild), 4-7 (moderate), and 8-10 (high). Data were analyzed using descriptive analysis. Wilcoxon rank-sum test measured the association of pain score with gender. Pain scales were correlated using Spearman correlation coefficient. Pain scale association with opioid treatment was tested via ordinal logistic regression controlling for gender, home opioid use, and if ED revisit was for pain.

\section{Results}

Average patient age was 49. Most patients were African American (68.4\%), male (51.2\%), and returned for pain $(67.0 \%)$. As continuous measures, both scales were positively correlated with each other $(\mathrm{p}<0.0001)$. Pain score severity categories were distributed differently between the two scales $(\mathrm{p}=0.0085)$, decreasing by $8 \%$ in patients reporting high pain severity when using DVPRS. For both scales, the proportion of patients ( 1 ) administered opioids ( $\mathrm{p}=0.0009$ and $\mathrm{p} \leqslant 0.0001$, respectively) and (2) discharged with opioids $(\mathrm{p}=0.0103$ and $\mathrm{p}=0.0417$, respectively) increased with pain severity. Discharge NRS $(\mathrm{p}=0.0001)(\mathrm{OR}=3.2$, $1.780-5.988)$ and DVPRS pain score categories $(\mathrm{p}<0.0001)(\mathrm{OR}=2.7,95 \% \mathrm{CI}=1.63-4.473)$ were associated with revisits for pain.

\section{Conclusions}

Our findings demonstrate a link between NRS and administration of opioid medications and suggest that DVPRS may better differentiate between moderate and high levels of pain in the ED setting.

Categories: Emergency Medicine, Pain Management, Substance Use and Addiction

Keywords: dvprs, nrs, pain scores, emergency department, misuse, abuse, opioid epidemic

\section{Introduction}

Over $70 \%$ of patients presenting to the emergency department (ED) arrive with a pain-related chief complaint $[1,2]$. Assessing pain severity can be elusive due to its subjective multifaceted nature and basis in self-report. Several pain severity scales exist in attempts to quantify and characterize patients' pain. One of the most widely used scales in medical practice is the unidimensional Numerical Rating Scale (NRS). Its use in assessment of pain intensity in acute to chronic pain has been reliable and validated in several populations and settings and correlates well with other pain scales employed in the assessment of pain intensity [2-7]. Features such as simplicity and rapidity of scoring and versatility in its mode of administration (including as a verbal assessment) explain why the NRS is a popular pain intensity scale in acute care settings like prehospital and the ED. However, unlike multidimensional pain scales, it does not factor the impact of pain on physical and emotional functioning. Patients using the NRS may exhibit a great degree of variability in interpreting and defining pain associated with each numerical value.

In contrast, the multidimensional Defense and Veterans Pain Rating Scale (DVPRS) combines functional 


\section{Cureus}

assessment with pain intensity, assigning descriptive language, color coding, and visuals of faces to each number on its pain scale, drawing from the previously validated Faces Pain Scale Revised (FPS-R) $[3,8,9]$. The construct validity, internal consistency reliability, and test-retest reliability of the DVPRS have been established within military inpatient and outpatient populations $[10,11]$. However, its application within the civilian emergency department setting has not been described within the literature.

Implementation of NRS in the ED setting has been noted to increase the frequency of analgesic administration, with higher pain scores exhibiting increased likelihood of analgesia use [12]. While it is important to address pain, failure to fully characterize a patient's pain or sole reliance on NRS to guide treatment has been associated with increased incidence of opioid adverse drug reactions, including oversedation [13]. While the NRS has been compared against other pain instruments, such as the Visual Analog Scale (VAS) and the Verbal Rating Scale (VRS), to our knowledge the correlation of the NRS with the pain intensity component of the DVPRS has not yet been studied within the literature [7,14]. To determine the relationship between DVPRS and NRS as measures of pain intensity, we compared pain scores to sociodemographics, opioid treatment data, and if ED return visit was for pain in patients revisiting the ED within 30 days of an index visit. Secondary outcomes included association of opioid use with DVPRS and NRS absolute and categorical ratings.

\section{Materials And Methods}

\section{Study setting and enrollment}

Study enrollment occurred in the ED (annual patient volume over 70,000) of an urban safety-net hospital system. The average patient presenting to our ED is 50 years old, $51 \%$ are African American, $50 \%$ are male, and $25 \%$ are on Medicare. This study was a secondary analysis of data collected from the University of Florida Institutional Review Board-approved prospective observational study of 389 patients $\geqslant 18$ years of age returning to the ED within 30 days of an initial (index) visit (Figure 1). Full study details have been published previously $[15,16]$. Briefly, all community-dwelling adult patients reporting to our ED and able to provide consent were eligible for inclusion. Patients not meeting these criteria and those instructed to return to the ED within a set time frame were excluded (e.g., scheduled wound checks or return for suture removal). Patients were enrolled using systematic time block sampling to mimic ED utilization rates by ED shift times based on historical data from our institution.

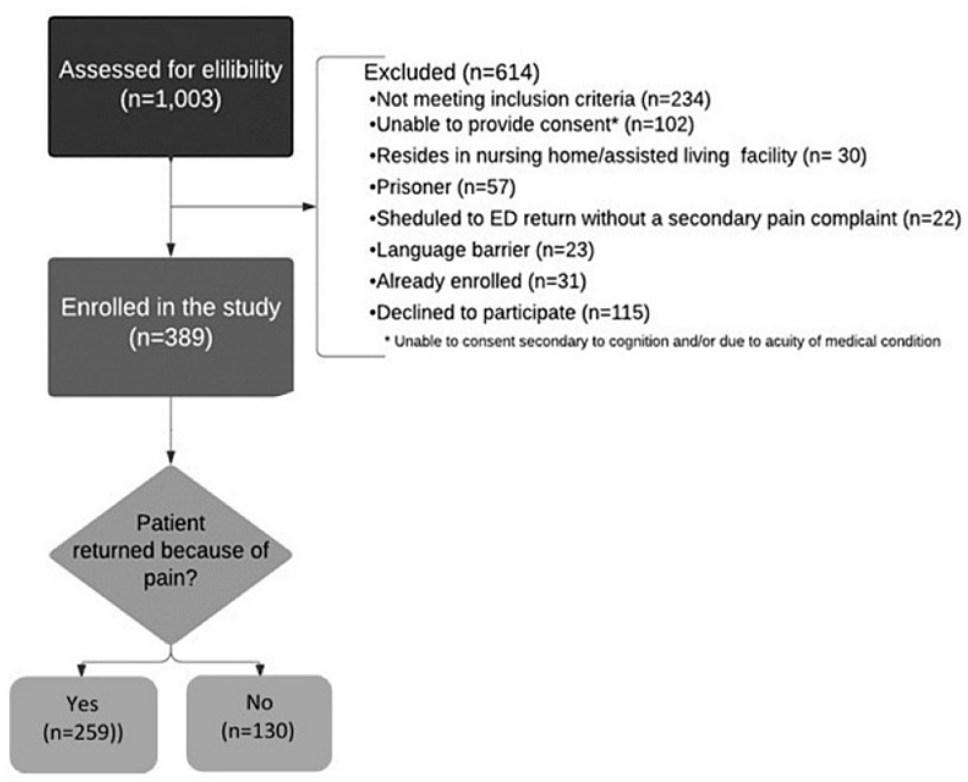

FIGURE 1: Patient Enrollment Schematic

\section{Variables collected}

Sociodemographic variables, health literacy level, comorbidities, medication history, NRS and DVPRS pain scores, receipt of an opioid prescription, and reason for ED return within 30 days after discharge were collected from patient interviews and chart review. The Rapid Estimate of Adult Literacy in Medicine (REALM) was used to assess reading health literacy level (scores 0-18=third grade and below; 19-44=fourth to sixth grade; $45-60=$ seventh to eighth grade; and 61-66=high school) [17]. Pain scores were classified as follows: 0-3 (mild), 4-7 (moderate), and 8-10 (high). 


\section{Cureus}

\section{Data analysis}

Descriptive analysis was used to characterize demographics and clinical characteristics of the included study patients. Spearman correlation coefficient was used to describe the correlation of the pain scales with each other since they were not normally distributed. For the association of pain scale scores with male versus female gender, Wilcoxon rank sum tests were used. To test the association of the different pain scores with 1) ED opioid medication administration and 2) ED prescription of an opioid, we first attempted a linear regression. However, traditional transformations were unsuccessful in achieving a normal distribution for the pain scores. Therefore, we converted each pain score into an ordinal variable, with $0-3$ equaling mild pain, 4-7 moderate pain, and 8-10 severe pain. We then performed an ordinal logistic regression controlling for gender, prior home opioid prescription, and if the ED revisit was for pain. All analysis was performed with SAS (Cary, NC), v9.4.

\section{Results}

\section{Study population characteristics}

Tables 1, 2 display the study population characteristics. The mean patient age was 49 . The mean health literacy level was seventh- to eighth-grade reading level. Of the 389 patients enrolled, 67\% (259) of patients returned because of pain, 51\% (129) for acute pain, and 18\% (48) for chronic pain. In the group of patients returning to the ED for pain, $46 \%$ (119) reported use of opioid medications at home. 


\section{Cureus}

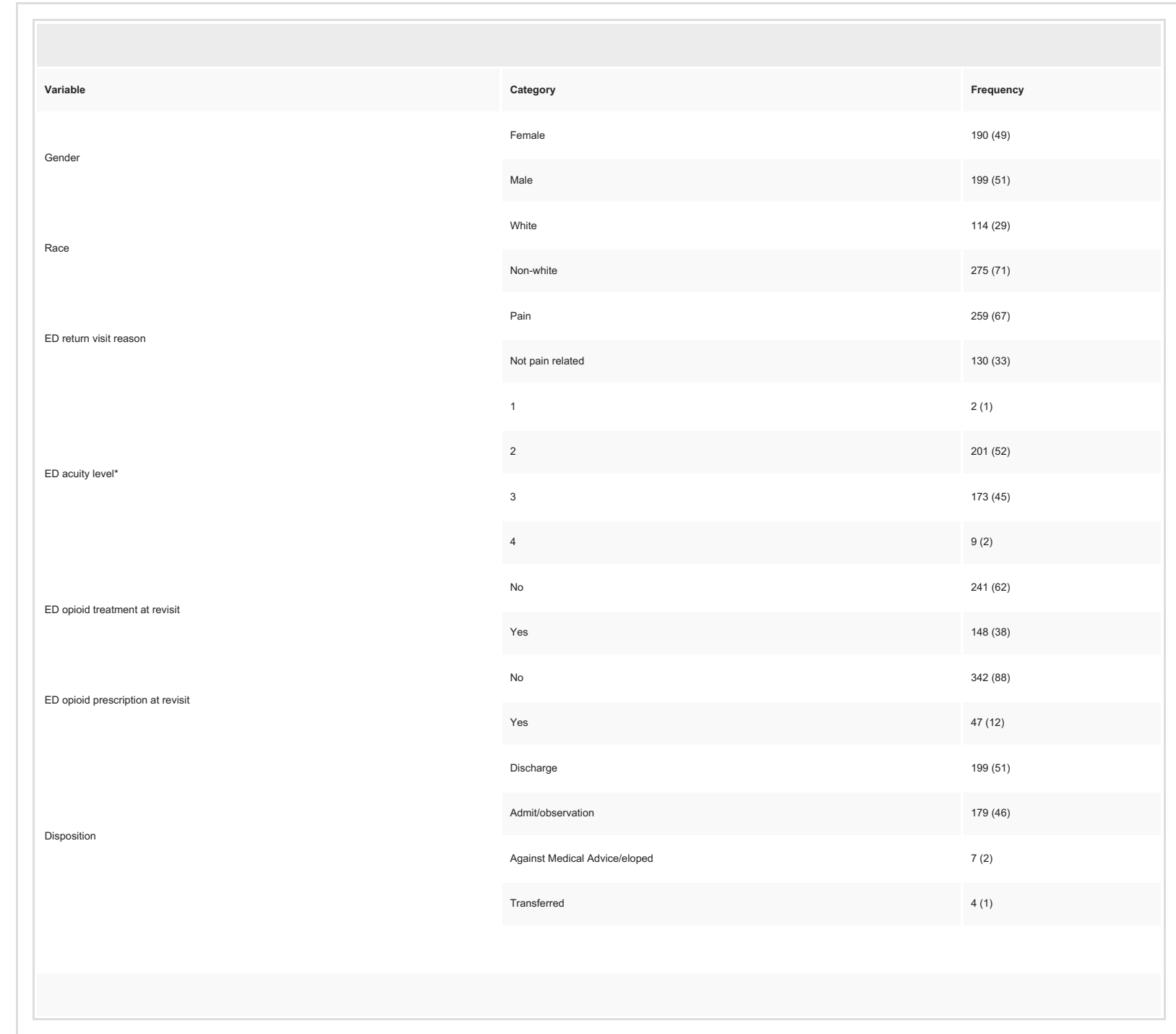

\section{TABLE 1: Characteristics of Study Population (Categorical Variables)}

*Emergency Severity Index Triage Tool: acuity level 1: immediate; acuity level 2: emergent; acuity level 3: urgent; acuity level 4: semiurgent; and acuity level 5: nonurgent. 


\section{Cureus}

\begin{tabular}{|c|c|c|c|c|c|c|}
\hline Variable & $\mathrm{N}$ & Mean & Standard Deviation & Median & First Quartile & Third Quartile \\
\hline Age & 389 & 49.4 & 15.4 & 51.8 & 37.8 & 60.1 \\
\hline Health ilitracy (REALM Score) & 354 & 52.6 & 17.2 & 60 & 49 & 64 \\
\hline DVPRS pain score & 373 & 6.6 & 3.2 & 8 & 5 & 9 \\
\hline NRS pain score & 386 & 6.4 & 3.7 & 8 & 4 & 10 \\
\hline Total number of ED visits in 30 days & 389 & 2.9 & 1.7 & 2 & 2 & 3 \\
\hline
\end{tabular}

\section{TABLE 2: Characteristics of Study Population (Continuous Variables)}

DVPRS: Defense and Veterans Pain Rating Scale, NRS: Numeric Rating Scale, REALM: Rapid Estimate of Adult Literacy in Medicine.

\section{Comparison of pain scales at ED revisit: univariate analysis}

As continuous measures, the NRS and DVPRS were positively correlated with each other $(\mathrm{p}<0.0001)$. Both the NRS and DVPRS were negatively correlated with age ( $\mathrm{p}=0.0004$ and $\mathrm{p}=0.0071$, respectively). Pain scale severity was not associated with health literacy for either scale. Pain score severity categories were distributed differently between the two scales, particularly for the moderate and high pain severity groups $(\mathrm{p}=0.0085$, Table 3$)$.

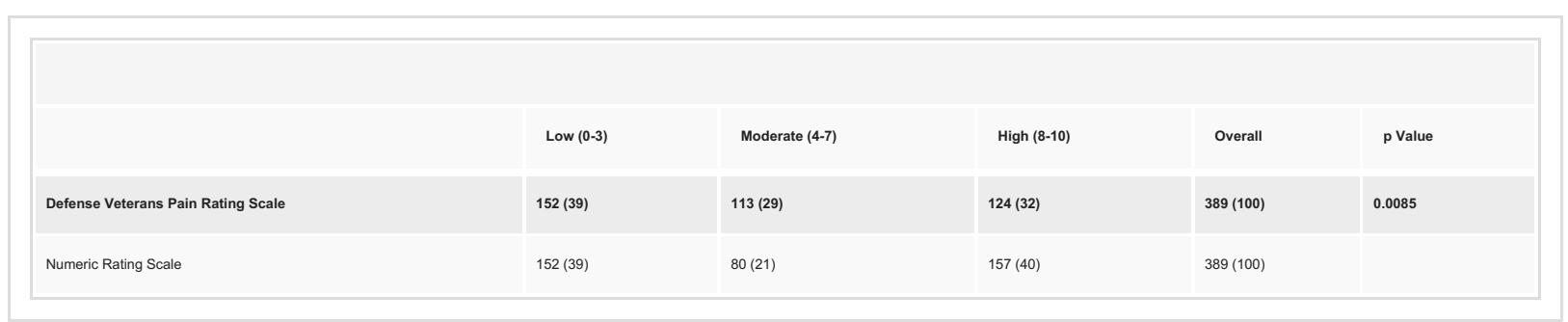

TABLE 3: Comparison of Pain Scales by Pain Category Severity

Table 4 displays the relationship between categorical variables and pain severity categories for each pain scale at the time of ED revisit. Pain category severity was distributed differently by gender for the DVPRS but not for the NRS ( $\mathrm{p}=0.02$ and $\mathrm{p}=0.57$, respectively). The proportion of patients who received opioid treatment in the ED increased as NRS and DVPRS pain category severity increased from low to high ( $p \leqslant 0.0001$ and $\mathrm{p}=0.0009$, respectively). DVPRS scores were distributed differently in patients discharged with prescription opioids from the $\mathrm{ED}(\mathrm{p}=0.0417)$. The proportion of patients discharged with an ED opioid prescription increased as NRS pain category severity increased $(\mathrm{p}=0.0103)$. The proportion of patients administered opioid medications in the ED increased as the NRS pain category severity worsened at the time of ED discharge $(p \leqslant 0.0001)$. The proportion of patients discharged with an opioid prescription also increased as the NRS pain category severity worsened at the time of ED discharge. 


\begin{tabular}{|c|c|c|c|c|c|c|c|c|c|c|c|}
\hline \multirow[b]{2}{*}{ Variable (n, \%) } & \multirow[b]{2}{*}{ Category } & \multicolumn{5}{|c|}{ DVPRS Category } & \multicolumn{5}{|c|}{ NRS Category } \\
\hline & & Low & Moderate & High & Total & $\mathrm{p}$ Value & Low & Moderate & High & Total & $\mathrm{p}$ Value \\
\hline \multirow{2}{*}{ Gender" } & Female & $36(20)$ & $44(24)$ & $104(57)$ & $184(49)$ & \multirow{2}{*}{0.0200} & $50(26)$ & $32(17)$ & $108(57)$ & $190(49)$ & \multirow{2}{*}{0.5700} \\
\hline & Male & $29(15)$ & $70(37)$ & $90(48)$ & $189(51)$ & & $51(26)$ & $41(21)$ & 104 (53) & $196(51)$ & \\
\hline \multirow{2}{*}{ Race } & White & $15(14)$ & $40(38)$ & $51(48)$ & $106(28)$ & \multirow{2}{*}{0.1500} & $25(22)$ & $28(25)$ & $61(53)$ & $114(30)$ & \multirow{2}{*}{0.1400} \\
\hline & Non-white & $50(19)$ & $74(28)$ & $143(54)$ & $267(72)$ & & $76(28)$ & $45(16)$ & $151(56)$ & $272(70)$ & \\
\hline \multirow{2}{*}{ ED opioid treatment } & No & $108(45)$ & $72(30)$ & $61(25)$ & $241(62)$ & \multirow{2}{*}{0.0009} & $122(51)$ & $60(25)$ & $59(24)$ & 241 (62) & \multirow{2}{*}{$<0.000$} \\
\hline & Yes & $44(30)$ & $41(28)$ & $63(42)$ & $148(38)$ & & $30(20)$ & 20 (14) & $98(66)$ & $148(38)$ & \\
\hline \multirow{2}{*}{ ED opioid prescription } & No & $138(40)$ & $92(27)$ & $112(33)$ & $342(88)$ & \multirow{2}{*}{0.0417} & $143(42)$ & $66(19)$ & $133(39)$ & $342(88)$ & \multirow{2}{*}{0.0103} \\
\hline & Yes & $14(30)$ & $21(45)$ & $12(25)$ & $47(12)$ & & $9(19)$ & $14(30)$ & $24(51)$ & $47(12)$ & \\
\hline \multirow{3}{*}{ Disposition } & Discharge & $29(15)$ & $68(34)$ & $101(51)$ & $198(53)$ & \multirow{3}{*}{0.3890} & $40(20)$ & $39(20)$ & $118(60)$ & $197(52)$ & \multirow{3}{*}{0.8290} \\
\hline & Admit & $35(21)$ & $44(26)$ & $89(53)$ & $168(45)$ & & $58(33)$ & $32(18)$ & $88(49)$ & $178(46)$ & \\
\hline & AMA+/ eloped & $1(14)$ & $2(29)$ & $4(57)$ & $7(2)$ & & $1(14)$ & $1(14)$ & $5(72)$ & $7(2)$ & \\
\hline
\end{tabular}

\section{TABLE 4: Comparison of Categorical Variables by Pain Scale}

All tests were done using Chi-Squared unless indicated by *, which was analyzed using Wilcoxon Rank Sum.

AMA: Against Medical Advice, DVPRS: Defense Veterans Pain Rating Scale, NRS: Numeric Rating Scale.

\section{Comparison of pain scales at ED revisit: multivariable analysis}

Increasing NRS pain category severity at triage predicted ED opioid administration $(\mathrm{p}<0.0001)(\mathrm{OR}=2.7$, 1.639-4.339). Increasing NRS pain category severity at ED triage was associated with prior home opioid prescriptions $(\mathrm{p}=0.03)(\mathrm{OR}=1.7,1.04-2.7)$ and if the $\mathrm{ED}$ return visit was for pain $(\mathrm{p}<0.001)(\mathrm{OR}=4.8,2.9-8.0)$. Increasing NRS pain category severity at the time of ED discharge was associated with whether the ED revisit was because of pain $(\mathrm{p}=0.0001)(\mathrm{OR}=3.2,1.780-5.988)$. Increasing DVPRS pain category severity was also significantly associated with ED revisits for pain in the multivariable model $(\mathrm{p}<0.0001)(\mathrm{OR}=3.101,95 \%$ $\mathrm{CI}=1.926-4.992)$. Neither scale predicted whether a patient received a prescription for an opioid medication at discharge.

\section{Discussion}

In comparing the pain intensity component of the DVPRS to that of the NRS, we found several similarities between the two scales. Both scales had a similar relationship with age, and they both predicted ED returns for pain as pain category severity increased. Also, neither scale predicted receipt of an opioid prescription at the time of ED discharge in the multivariable model.

Although we found that the two scales were positively correlated with one another when they were used as continuous measures and when scores were grouped into severity categories (low, moderate, and high), we were better able to characterize the relationship between the two scales. First, the proportion of patients in the low pain group was the same for both pain scales. These patients reported a pain score between 0 and 3 regardless of which pain scale was used. Second, the proportion of patients in the moderate and high pain groups changed depending on the pain scale used. The proportion of patients reporting high pain scores decreased from $40 \%$ to $32 \%$ when using the DVPRS. There was also a corresponding increase of $21 \%$ to $29 \%$ in the proportion reporting moderate pain when using the DVPRS. This indicates that the DVPRS may discriminate between moderate and high levels of pain more effectively than the NRS.

We also found that increasing NRS pain category severity was associated with a three-fold higher likelihood of receiving opioid treatment in the ED. The NRS predicting ED administration of opioid medications in our patient sample demonstrates the power of pain scales to influence pain management, specifically opioid administration, in acute care settings like the ED. Others have also reported associations between unidimensional pain scales and increased analgesia administration and opioid adverse events [14,15]. Our 
results of increasing NRS pain category severity predicting ED opioid administration and DVPRS differentiating between moderate and high levels of pain coupled together suggest that patients may receive fewer opioid medications in the ED if the DVPRS (and not NRS) is used to measure pain intensity. These findings should be confirmed in a larger patient population.

There are several limitations to note in this study. This study recruited patients from only one site. Additionally, $71 \%$ of our participants self-identified as non-white and most had limited health literacy. While these factors may limit the potential to apply our results to other ED populations, our findings are important in that they are derived from a largely minority and socially vulnerable patient population who tend to be underrepresented in the pain literature.

\section{Conclusions}

With this analysis, we demonstrated a clear link between NRS scores and administration of opioid medications. Additionally, our results suggest that the DVPRS may be better at differentiating between moderate and high levels of pain compared to the NRS in the ED setting. Further study should be conducted to confirm these findings and should be replicated in other ED populations, particularly in those who are underrepresented in the pain literature.

\section{Additional Information \\ Disclosures}

Human subjects: Consent was obtained or waived by all participants in this study. University of Florida issued approval IRB201601567. We have received Institutional Review Board (IRB) approval to conduct the above-listed research project. Approval of this project was granted on $9 / 27 / 2016$ by IRB-03 . This study is approved as expedited because it poses minimal risk and is approved under the following expedited category/categories: 7. Research on individual or group characteristics or behavior (including, but not limited to, research on perception, cognition, motivation, identity, language, communication, cultural beliefs or practices, and social behavior) or research employing survey, interview, oral history, focus group, program evaluation, human factors evaluation, or quality assurance methodologies. [ Note: Some research in this category may be exempt from the HHS regulations for the protection of human subjects. (45 CFR 46.101(b)(2) and (b)(3).) This listing refers only to research that is not exempt. ] . Animal subjects: All authors have confirmed that this study did not involve animal subjects or tissue. Conflicts of interest: In compliance with the ICMJE uniform disclosure form, all authors declare the following: Payment/services info: Drs. Sophia Sheikh, Colleen Kalynych, and Phyllis Hendry received support from the Florida Medical Malpractice Joint Underwriter's Association Dr. Alvin E. Smith Safety of Healthcare Services Grant. Dr. Sophia Sheikh was also supported by the NIH/NIA-funded Jacksonville Aging Studies Center (JAX-ASCENT; R33AG05654). Dr. Ashley Norse was the recipient of a Dean's Fund for Research Award from the University of Florida College of Medicine - Jacksonville. . Financial relationships: All authors have declared that they have no financial relationships at present or within the previous three years with any organizations that might have an interest in the submitted work. Other relationships: All authors have declared that there are no other relationships or activities that could appear to have influenced the submitted work.

\section{Acknowledgements}

Authors would like to thank the study participants and research staff who made this work possible.

\section{References}

1. Tanabe P, Buschmann M: A prospective study of ED pain management practices and the patient's perspective. J Emerg Nurs. 1999, 25:171-7. 10.1016/s0099-1767(99)70200-x

2. Mura P, Serra E, Marinangeli F, et al.: Prospective study on prevalence, intensity, type, and therapy of acute pain in a second-level urban emergency department. J Pain Res. 2017, 10:2781-8. 10.2147/JPR.S137992

3. Bijur PE, Latimer CT, Gallagher EJ: Validation of a verbally administered numerical rating scale of acute pain for use in the emergency department. Acad Emerg Med. 2003, 10:390-2. 10.1111/j.15532712.2003.tb01355.x

4. Breivik EK, Björnsson GA, Skovlund E: A comparison of pain rating scales by sampling from clinical trial data. Clin J Pain. 2000, 16:22-8. 10.1097/00002508-200003000-00005

5. Jensen MP, Karoly P, Braver S: The measurement of clinical pain intensity: a comparison of six methods Pain. 1986, 27:117-26. 10.1016/0304-3959(86)90228-9

6. Jensen MP, Karoly P, O'Riordan EF, Bland F Jr, Burns RS: The subjective experience of acute pain. An assessment of the utility of 10 indices. Clin J Pain. 1989, 5:153-60. 10.1097/00002508-198906000-00005

7. Williamson A, Hoggart B: Pain: a review of three commonly used pain rating scales . J Clin Nurs. 2005, 14:798-804. 10.1111/j.1365-2702.2005.01121.x

8. Bieri D, Reeve RA, Champion DG, Addicoat L, Ziegler JB: The Faces Pain Scale for the self-assessment of the severity of pain experienced by children: development, initial validation, and preliminary investigation for ratio scale properties. Pain. 1990, 41:139-50. 10.1016/0304-3959(90)90018-9

9. Polomano RC, Galloway KT, Kent ML, Brandon-Edwards H, Kwon KN, Morales C, Buckenmaier C 3rd: Psychometric testing of the Defense and Veterans Pain Rating Scale (DVPRS): a new pain scale for military population. Pain Med. 2016, 17:1505-19. 10.1093/pm/pnw105 


\section{Cureus}

10. Buckenmaier CC 3rd, Galloway KT, Polomano RC, McDuffie M, Kwon N, Gallagher RM: Preliminary validation of the Defense and Veterans Pain Rating Scale (DVPRS) in a military population. Pain Med. 2013, 14:110-23. 10.1111/j.1526-4637.2012.01516.x

11. Nassif TH, Hull A, Holliday SB, Sullivan P, Sandbrink F: Concurrent validity of the Defense and Veterans Pain Rating Scale in VA outpatients. Pain Med. 2015, 16:2152-61. 10.1111/pme.12866

12. Nelson BP, Cohen D, Lander O, Crawford N, Viccellio AW, Singer AJ: Mandated pain scales improve frequency of ED analgesic administration. Am J Emerg Med. 2004, 22:582-5. 10.1016/j.ajem.2004.09.003

13. Vila H Jr, Smith RA, Augustyniak MJ, et al.: The efficacy and safety of pain management before and after implementation of hospital-wide pain management standards: is patient safety compromised by treatment based solely on numerical pain ratings?. Anesth Analg. 2005, 101:474-80. 10.1213/01.ANE.0000155970.45321.A8

14. Shafshak TS, Elnemr R: The Visual Analogue Scale versus Numerical Rating Scale in measuring pain severity and predicting disability in low back pain [PREPRINT]. J Clin Rheumatol. 2020, 10.1097/RHU.0000000000001320

15. Sheikh S, Booth-Norse A, Smotherman C, et al.: Predicting pain-related 30-day emergency department return visits in middle-aged and older adults. Pain Med. 2020, 21:2748-56. 10.1093/pm/pnaa213

16. Sheikh S, Booth-Norse A, Holden D, et al.: Opioid overdose risk in patients returning to the emergency department for pain [PREPRINT]. Pain Med. 2021, 10.1093/pm/pnab047

17. Davis TC, Crouch MA, Long SW, Jackson RH, Bates P, George RB, Bairnsfather LE: Rapid assessment of literacy levels of adult primary care patients. Fam Med. 1991, 23:433-5. 